\title{
Emergence of Hyalomma marginatum and Hyalomma rufipes adults revealed by citizen science tick monitoring in Hungary
}

\author{
Gabor Foldvari ${ }^{1}$, Éva Szabó${ }^{1}$, Gábor Endre Tóth ${ }^{2}$, Zsófia Lanszki², Brigitta Zana², Zsaklin \\ Varga $^{2}$, and Gabor Kemenesi ${ }^{2}$ \\ ${ }^{1}$ Okologiai Kutatokozpont \\ ${ }^{2}$ University of Pecs Biologia Intezet
}

February 24, 2022

\begin{abstract}
Hyalomma ticks are important vectors of Crimean-Congo Haemorrhagic Fever Virus (CCHFV) and other pathogens. They are frequently carried as immatures from Africa, the Middle East and Mediterranean areas to temperate Europe via migratory birds and emergence of its adults has been reported in many countries where it has so far been non-endemic. Our aim was to implement the first steps of the DAMA (Document, Assess, Monitor, Act) protocol by monitoring the potential arrival of adult Hyalomma ticks in Hungary applying citizen-science methods. Ticks were collected from April-December 2021 by asking volunteer participants through a self-made website to look for unusual hard ticks on themselves, their pets and livestock. Owing to the intensive media campaign, the project website had over 31 thousand visitors within seven months and 137 specimens and several hundreds of photos of hard ticks were submitted by citizen scientists from all over the country. Beside Ixodes ricinus, Dermacentor reticulatus, Dermacentor marginatus and Haemaphysalis inermis, a specimen from a dog was morphologically identified as a male Hyalomma marginatum and another removed from a cattle as a male Hyalomma rufipes. The dog and the cattle had never been abroad, they were approximately $280 \mathrm{~km}$ apart, thus the two Hyalomma observations can be considered as separate introductions. Amplification of the partial mitochondrial cytochrome C oxidase subunit I gene was successful for both specimens. Sequencing verified the previous morphological identification for both ticks. Based on the phylogenetic analyses the Hy. marginatum individual most likely belongs to the Eurasian population and the Hy. rufipes to a clade of mixed sequences from Europe and Africa. We summarize the scattered historical reports about the occurrence of Hyalomma ticks and CCHFV in Hungary. Our data highlight the effectiveness of citizens science programmes in the monitoring and risk assessment of CCHFV emergence and preparedness in our region.
\end{abstract}

Emergence of Hyalomma marginatum and Hyalomma rufipes adults revealed by citizen science tick monitoring in Hungary

Gábor Földvári ${ }^{1} *$, Éva Szabó ${ }^{1}$, Gábor Endre Tóth ${ }^{2,3}$, Zsófia Lanszki ${ }^{2,3}$, Brigitta Zana ${ }^{2,3}$, Zsaklin Varga ${ }^{2,3}$, Gábor Kemenesi ${ }^{2,3}$

${ }^{1}$ Institute of Evolution, Centre for Ecological Research, 1121 Budapest, Konkoly-Thege Miklós út 29-33.

${ }^{2}$ National Laboratory of Virology, Szentágothai Research Centre, University of Pécs, 7624 Pécs, Hungary

${ }^{3}$ Institute of Biology, Faculty of Sciences, University of Pécs, 7624 Pécs, Hungary

* Corresponding author FoldvariGabor@gmx.de

Summary

Hyalomma ticks are important vectors of Crimean-Congo Haemorrhagic Fever Virus (CCHFV) and other pathogens. They are frequently carried as immatures from Africa, the Middle East and Mediterranean 
areas to temperate Europe via migratory birds and emergence of its adults has been reported in many countries where it has so far been non-endemic. Our aim was to implement the first steps of the DAMA (Document, Assess, Monitor, Act) protocol by monitoring the potential arrival of adult Hyalomma ticks in Hungary applying citizen-science methods. Ticks were collected from April-December 2021 by asking volunteer participants through a self-made website to look for unusual hard ticks on themselves, their pets and livestock. Owing to the intensive media campaign, the project website had over 31 thousand visitors within seven months and 137 specimens and several hundreds of photos of hard ticks were submitted by citizen scientists from all over the country. Beside Ixodes ricinus, Dermacentor reticulatus, Dermacentor marginatus and Haemaphysalis inermis, a specimen from a dog was morphologically identified as a maleHyalomma marginatum and another removed from a cattle as a maleHyalomma rufipes. The dog and the cattle had never been abroad, they were approximately $280 \mathrm{~km}$ apart, thus the two Hyalommaobservations can be considered as separate introductions. Amplification of the partial mitochondrial cytochrome $\mathrm{C}$ oxidase subunit I gene was successful for both specimens. Sequencing verified the previous morphological identification for both ticks. Based on the phylogenetic analyses the Hy. marginatum individual most likely belongs to the Eurasian population and the Hy. rufipes to a clade of mixed sequences from Europe and Africa. We summarize the scattered historical reports about the occurrence of Hyalomma ticks and CCHFV in Hungary. Our data highlight the effectiveness of citizens science programmes in the monitoring and risk assessment of CCHFV emergence and preparedness in our region.

\section{Acknowledgements}

We are grateful to all citizen scientists who took part in the project and helped with submitting photos, ticks or data. We would like to acknowledge Kornél Baráth and Attila Ádám for providing theHyalomma specimens. We thank Zoltán Soltész for his advice on the citizen science project and the excellent work of Eszter Draskóczy who played a crucial role in reaching society through the appropriate media appearances. The help of Sonia A. Olmeda and Félix Valcárcel in confirming our morphological identification of the two Hyalommaspecimens is much appreciated.

Keywords: Crimean-Congo Haemorrhagic Fever, DAMA protocol, emerging infectious diseases, imported ticks, Hungary, Hyalomma adult

\section{Background}

Emergence of pathogens and vectors in new hosts and new geographical areas poses great threats for public and veterinary health (Jones et al., 2008). Ticks are responsible for the spread of numerous pathogens worldwide and species belonging to the genus Hyalomma have been shown to have a particular epidemiologic role (Estrada-Peña, DAmico, \& Fernández-Ruiz, 2020; Hubálek, Sedláček, Estrada-Peña, Vojtíšek, \& Rudolf, 2020). The tick species Hyalomma marginatum and Hyalomma rufipes are the main vectors of Crimean-Congo haemorrhagic fever virus (CCHFV). This is a negative-sense single-stranded RNA virus belonging to the Nairoviridae family (order Bunyavirales) . It can cause debilitating human disease with no available casual treatment or vaccine and has a case fatality rate up to $30 \%$ (Bente et al., 2013). The public health significance of Crimean-Congo haemorrhagic fever is demonstrated by the fact that this infection is geographically the most widespread tick-transmitted viral disease in humans. It is found in the southern parts of Asia, the Middle East, Europe and Africa. The emergence of the virus is mainly determined by the distribution of Hyalomma ticks being responsible for the spread of the disease agent (Bente et al., 2013).

Hyalomma marginatum and Hy. rufipes are two-host ticks (Estrada-Peña, Mihalca, \& Petney, 2018). The larva moults into a nymph on the same first host, feeding on that animal at both stages of development. For these two Hyalomma species, the first host is very often a bird. The fact that they do not change host before becoming a nymph greatly contributes to the successful geographical spread of these ticks, as it allows them to travel longer distances by attaching to the same migratory bird host. The adults moult from the engorged nymphs dropping off the first host and actively look for the second host, which is usually a large wild or domesticated mammal or human (Valcárcel et al., 2020; Walker et al., 2003).

Although the yearly arrival of immature Hyalomma ticks with migratory birds in temperate Europe has been 
a frequent phenomenon (Capek et al., 2014), the emergence of its adults has only been detected in higher numbers and wider distribution recently. Adults of Hy. marginatum were found in Slovakia (Nosek, Kožuch, \& Lysý, 1982) and Austria (Duscher et al., 2018), Hyalomma rufipes adults were found in Hungary (S Hornok \& Horváth, 2012). The adults of both tick species have recently been detected the Czech Republic (Hubálek et al., 2020; Lesiczka et al., 2022; Rudolf et al., 2021) Germany (Chitimia-Dobler et al., 2019), Sweden (Grandi et al., 2020), United Kingdom (Hansford et al., 2019; McGinley et al., 2021) and the Netherlands (Uiterwijk et al., 2021).

Citizen science, or community research, is a method that is gaining in popularity and involvement in many research fields today. The basic idea is that the data are collected or generated with the help of non-researchers but are analysed by researchers specializing in the given topic (Tran et al., 2021). Citizen science has many benefits, both for the researchers who evaluate the data and for the citizens who collect them. With the help of citizen science, for example, professionals have access to the data they need without having to pay for the work of hundreds of civilian researchers involved in the project, and the travel, accommodation and education that may be associated with it. It is also a great advantage that a project can be carried out over a large geographical area for many years (MacPhail \& Colla, 2020). This approach has been successfully used in searching for the emergence of Hyalomma adults in several European countries (Grandi et al., 2020; Lesiczka et al., 2022; Uiterwijk et al., 2021).

In Hungary, we have limited published information about the occurrence of Hyalomma ticks. An engorged nymph of Hy. marginatum has been reported from a hedgehog (Erinaceus roumanicus) living in a Budapest city park (G Földvári et al., 2011) and two larvae and a nymph were identified from robins (Erithacus rubecula ) (S Hornok et al., 2013) sampled to the south of the capital. Three Hy. rufipesnymphs were reported from a common whitethroat (Sylvia communis ) in another study (S. Hornok et al., 2016). The presence of antibodies against CCHFV has also been detected in Hungarian hares (Lepus europaeus ) (Németh et al., 2013), rodents (Földes et al., 2019) and recently also in humans (Magyar et al., 2021).

Based on these previous findings, the aim of our research was to obtain a comprehensive, nationwide picture of the occurrence of adultHyalomma ticks in Hungary through a citizen science approach. This kind of documentation is of great epidemiological importance because this is the first step for the prevention of emerging pathogens according to the DAMA (Document, Assess, Monitor, Act) protocol (Brooks et al., 2014). Based on this, we plan to continuously document the appearance of pathogens, their vectors and reservoirs hosts, then, after the appropriate scientific analyses (Assess), we will be able to identify the organisms that pose a threat to us and manage their targeted surveillance (Monitor). Thus, it will be possible to make adequate proposals to decision makers to take appropriate preventive measures (Act) (Brooks, Hoberg, \& Boeger, 2019).

\section{Materials and methods}

\section{Tick and data collection}

Ticks were collected from April-December 2021 within the tick monitoring project https://www.kullancsfigyelo.hu, a citizen-science project aimed at monitoring the emergence and possible establishment ofHyalomma ticks in Hungary. We were asking participants through the self-made website to look for unusual hard ticks on themselves, their pets and their livestock. We called attention to specimens larger than usual with visible stripes on their legs and with apparently quick movement. To enhance visibility, the project was intensively communicated in local and national on-line media outlets, local and national television and radio channels and social media platforms.

Ticks were reported either by high-quality images sent by e-mail or physically delivered to the Institute of Evolution, Centre for Ecological Research, personally or by mail. Information about safe removal, storage and postage of tick specimens was provided on the project website.

\section{Morphological identification of ticks}

Ticks received by mail were kept at $4^{\circ} \mathrm{C}$ and examined alive. Specimens were identified with a Nikon 
SMZ800N stereomicroscope using appropriate morphological characters and identification keys (EstradaPeña et al., 2018; Walker et al., 2003). The species, sex, developmental stage, engorgement state, host and place of origin of the ticks were recorded.

\section{Molecular identification of Hyalomma ticks}

Hyalomma specimens were homogenised with Bertin Minilys (Bertin Instruments, Montigny-le-Bretonneux, France) after 3 times freeze-thawing in liquid nitrogen. We used multiple beads in various sizes with the addition of quartz sand after the freeze-thaw cycles and before proceeding to . .Samples were sequenced at a commercial centre (Eurofins Genomics, Ebersberg, Germany).

Sequence data were subjected for phylogenetic analysis, using the MEGA11 (Tamura, Stecher, \& Kumar, 2021). The visualisation of final data was performed with iTOL v6 online tool.

\section{Results}

This tick monitoring project is still ongoing but the data for the present study are from the period between 28 April and 7 December 2021. During this time interval, the websitehttps://www.kullancsfigyelo.hu/had 31325 individual visitors. A total of 137 hard tick specimens and several hundreds of photos were received. Some other arthropods, e.g. soft ticks, spiders, bugs and a beetle were also sent by participants as a by-catch. Most of the ticks were identified as Ixodes ricinus, but Dermacentor reticulatus, Dermacentor marginatus and Haemaphysalis inermis also occurred (Table 1.). Some participants sent only photos from specimens, in these cases the identification was usually possible only to the genus level.

Two specimens were morphologically identified as Hyalomma ticks. The first Hyalomma was discovered in a

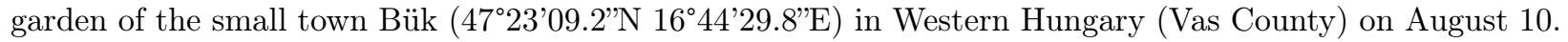
The tick was crawling on a dog's leg when the submitter noticed it. This tick was morphologically identified as a maleHyalomma marginatum. The second specimen was found feeding on a cattle at a farm near Kiskunmajsa $\left(46^{\circ} 26^{\prime} 09.6^{\prime \prime} \mathrm{N} 19^{\circ} 41^{\prime} 13.4^{\prime \prime E}\right)$ in South-Eastern Hungary (Bács-Kiskun County) on September 10. This individual was morphologically identified as a Hyalomma rufipesmale. The dog and the cattle had never been abroad. Following morphological identification, Hyalomma ticks were stored in liquid nitrogen until molecular analyses. Amplification of the COX1 gene was successful for both specimens. Sequencing verified the previous morphological identification as Hy. marginatum for the first specimen. Based on the phylogenetic analysis with cognate sequences this individual most likely belongs to the Eurasian population (Figure 1.). Sequencing of the COX1 gene fragment of the second specimen confirmedHy. rufipes with the highest sequence similarity to a clade of mixed sequences from Europe and Africa. (Figure 2.)

Tick specimens were sent by citizen scientists from all over the country, the largest number from Budapest and its surroundings. The least data were received from the south-east of the country (Figure 3). Ticks were either collected from the environment or from hosts. Most ticks were removed by citizen scientists from humans, with a total of 45 such cases. The second most common hosts were dogs with 18 submissions (including the Hy. marginatum specimen) and then cats with seven. A single specimen was received from a cattle, this was the animal in which the Hy. rufipes was discovered.

\section{Discussion}

Within seven months from its start, our tick monitoring citizen science research succeeded to reveal the emergence of two adult Hyalommatick species in two different locations of Hungary. Our project highlights the importance of public relations in the success of a citizen science project. We could reach relatively high visibility (over 31000 individual website visitors) within seven months (data from Google Analytics not shown). The highest number of daily visitors, 5453 could be reached after an extensive media release of our project. Participating civilians also benefit from initiatives that use citizen science. First, in the optimal case, all such research is intended to serve the interests of society directly or indirectly, so the interest of civilian researchers is the success of the program. In addition, the increased social awareness caused by various citizen science projects is a positive side effect. Due to their involvement in the survey, participants pay more attention to nature and to monitoring non-endemic tick species in their environment and the relationship 
between participating citizens, scientists and public health agencies may also become closer. Our participants received reply from us regarding their questions and samples within 24 hours. This was important because some secondary media appearances, emphasizing the seriousness of Crimean-Congo haemorrhagic fever, were frightening some of our responders, and in most of the cases they were comforted by the fact that the specimen they found was not a Hyalomma tick.

In addition to the advantages, we must not forget about the limitations and disadvantages of the method. For example, at the beginning of any program, it is important to pay close attention to accurately describing the information requested from the public, failing which it can be very time-consuming to select relevant data from the set of information received. It may also be the case that, due to the incorrect or unverifiable data received, the decision-making bodies may not take into account the results of such a project. It is particularly difficult to filter out or prevent the creation of such erroneous data, perhaps because citizen science is still often characterized by a lack of universal, reproducible methods. The introduction and strict adherence to these methods can easily dampen the enthusiasm of the participating civilians, which is why the high level of attention in project design is important (MacPhail \& Colla, 2020).

The form of the requested data may also be crucial for the preparation of the research. In monitoring the distribution of tick species, where, for example, citizen scientists may try to identify an animal found, the participants have three ways to submit the requested information. They can only send the name of the species identified, the photographic documentation of the individual, or the specimen itself. All three options have advantages and disadvantages, the first case favours large amounts of incoming data, but there is no way to verify them. In the second case, a lot depends on whether the person making the shot is aware of what morphological characters make the identification possible, e.g., from what angle and at what resolution it is worth taking pictures. The exact identification is most secure when citizen scientists send the specimen to be identified by professionals. We advised the participants to do so, however, in addition to being the most time consuming, this case raises other issues. Submission might involve the destruction of the specimen, and on the other hand, it increases the risk of human-tick contact (Eisen \& Eisen, n.d.). Therefore, we particularly made our participants aware of the risks and suggested the safe removal, storage and postage of tick specimens.

The most commonly submitted tick species was $I$. ricinus. This is in line with the widespread distribution of this species, however, it also shows that participants do frequently not recognise the most common tick species. Dermacentor ticks were also submitted in large numbers. This is again partly due to the common occurrence of this tick species throughout the country (G. Földvári et al., 2007), however the on-line media also biased the study in this aspect. Unfortunately, several news portals used images that featured a Dermacentorspecies instead of a Hyalomma tick. As a consequence, many citizen scientists, especially dog owners, sent Dermacentorspecimens that are larger and quicker compared to I. ricinus and, in addition, matched the mistakenly included photograph of some online portals. We archived all tick specimens and data for further analyses, thus this by-catch might provide important epidemiological data in the future.

Mitochondrial gene-based genetic analysis is highly important to reveal dispersal patterns and introduction routes of Hyalomma ticks (Capek et al., 2014; Wallménius et al., 2014). Novel mitochondrial sequence data for Hyalomma spp. is increasingly reported (Ciloglu et al., 2021; Lang, Shan, Zhang, Liu, \& Wang, 2022). The importance of providing sequence data of these vector organisms is getting more relevant in the light of disease emergence, especially the geographic expansion of Crimean-Congo Haemorrhagic Fever virus during the last decades (Akyildiz, Bente, Keles, Vatansever, \& Kar, 2021; Arteaga et al., 2021; Moraga-Fernández et al., 2021; Sánchez-Seco et al., n.d.). We further highlight the importance of sequence data by integrating this activity into the citizens science programme and also into the risk assessment of CCHFV emergence and preparedness in our region. The two sites were Hyalomma ticks were found in our study are approximately 280 kilometres apart, with the first specimen discovered on August 10 and the second on September 10, so exactly one month apart. Due to the large geographical distance and the time difference, the two specimens must have arrived in the country separately as a result of two separate introductions. Although much more published sequence data would be necessary, we suspect that the probable source of introduction was a Eurasian 
population for the detected Hy. marginatum and a population along the Africa-Europe bird migration route for Hy. rufipes .

Another important issue is whether the specimens found entered the country in the same year, e.g. as engorged nymphs dropping off from migratory birds, or overwintered before finding them in the next year, or even already hatched here as larvae in the previous year. The latter possibility is of greatest concern because it would indicate the establishment of a local population, making this species able to successfully complete its entire life cycle in Hungary. This seems unlikely currently, because a critical number of conspecificHyalomma adults would be needed to yield egg-laying fertilized females. The moulting into adult in the year of drop-off, among many other factors, is affected mainly by temperature. On the one hand, hot springs are preferred by Hyalomma ticks, where the average daily temperature above $8^{\circ} \mathrm{C}$ for $\mathrm{Hy}$. marginatum ticks can be determined as a criterion for moulting from nymph to adult (Gale et al., 2012). Due to the colder-thanaverage April weather in Hungary in 2021, this was only achieved in mid-May (National Meteorological Service). Only adults found before this could have unambiguously overwintered in the country, giving the opportunity to develop a local population. However, during our program, both specimens of Hyalomma were discovered well after May, so these adults were presumably introduced the same year. Our tick monitoring program was not running during the spring, and there is a possibility for overwintering adult Hyalomma in Hungary, as it has recently been shown in the Czech Republic (Rudolf et al., 2021). Unfed adult Hyalomma ticks tolerate the cold months well, with the most critical period being late summer and autumn due to the heat sensitivity of engorged nymphs (Uiterwijk et al., 2021; Valcárcel et al., 2020). Thus, it is not the cold winter but the cold autumn that is critical in shaping the geographical spread of Hyalomma species. Climate change might easily enhance establishment of new local Hyalomma populations with elevating autumn temperatures in temperate Europe as exemplified by the rising presence of the tick Hy. marginatum in Southern France (Vial et al., 2016). Once imported, establishment of Hy. marginatum on common local hosts, wild and domesticated mammals and even urban ones like hedgehog has been shown in Hungary ( $\mathrm{G}$ Földvári et al., 2011) and also recently in Bulgaria (Arnaudov, Mikov, \& Georgiev, 2022).

The ultimate reason for monitoring the emergence of adult Hyalomma ticks is the possible introduction of tickborne pathogens, most importantly Crimean-Congo Haemorrhagic Fever Virus. There are scattered historical data about the occurrence of Hyalomma ticks and CCHFV in Hungary as summarised in Figure 4. Immature Hy. marginatum from a hedgehog in a city park (G Földvári et al., 2011), immature Hy. rufipes and Hy. marginatum from songbirds (S Hornok et al., 2013; S. Hornok et al., 2016) and two adult Hy. rufipes from cattle were previously detected in the country. Reported occurrence of CCHFV spans from isolation from Ixodes ricinus ticks (Molnár, 1982), to seropositive cattle, sheep (S. Horváth, 1974), brown hares (Németh et al., 2013) and wild rodents (Földes et al., 2019). Most interestingly, humans seropositive for CCHFV were reported well before the first observation of adult Hyalomma ticks (L. B. Horváth, 1976). There was also a reported human case of CCHF with unknown origin in 2004 (Országos Epidemiológiai Központ, 2008) and CCHFV seropositives were detected in 12 healthy blood donors collected between 2008-2017 (Magyar et al., 2021). All these data indicate that CCHFV might be transmitted by tick species other than Hyalomma spp. and that the earlier emergence of adult Hyalomma ticks might have been overlooked in Hungary. In any case, we will continue with the citizen science based systematic monitoring of Hyalomma ticks in the future. We also plan to analyse these and future Hyalomma specimens for the occurrence of CCHFV and other pathogens.

\section{Conclusions}

In the course of our research, we examined the occurrence of non-endemicHyalomma ticks in Hungary using the citizen science method. The use of community research was successful in terms of both website traffic and number of submitters. Participants reacted positively, people took the program noticeably seriously. Due to the importance of the topic and the good reception, our goal is to continue the research next spring, so the first years' experience is also useful for fine-tuning the project. One striking result of our research was that one of the two Hyalomma found was discovered in the only cattle involved in our research. Hyalomma often parasitize large domesticated mammals in their adult stages, as was previously known (S Hornok 
\& Horváth, 2012; Uiterwijk et al., 2021). It would therefore be worthwhile next year for our program to target inspections of establishments affected in this respect. This could be done, for example, by finding journals, online platforms and social media groups for animal husbandry, horse riding, or by looking for farms specializing in these. Data were received from across the country, but most submissions are clearly from the capital or a narrower neighbourhood. In the future, greater emphasis should be placed on rural areas, especially livestock farms, villages and towns. As media presence has proven to be key to our research, in the future we will try to focus on the print and electronic media presence also in rural areas.

In addition, it would be worthwhile to launch a media campaign for the project as early as the end of the winter, ensuring that people take pictures of the ticks already discovered in the spring and send them via mail. This would be important because in such cases, there would probably only be individuals of the potentially overwintering Hyalomma ticks in the country, so such an observation would indicate that the group may be able to form local populations in the future. It is of utmost importance to continue systematic monitoring ofHyalomma ticks in the future. We will analyse these and futureHyalomma specimens for the occurrence of CCHFV and other pathogens to be able to monitor and prepare for the introduction of transboundary and emerging diseases.

\section{Conflict of interest}

The authors declare that they have no conflict of interest.

\section{Author contribution}

GF conceptualized, designed and coordinated the study and drafted the original manuscript. GF and ÉSz coordinated the citizen science project, morphologically identified ticks. ÉSz designed and managed the project websitehttps://www.kullancsfigyelo.hu/. GET, ZSL, ZSV performed laboratory work and sequencing. BZ, GK performed phylogenetic analysis. ZSL did data visualization. GK supervised the molecular and sequencing works. GET and GK edited and reviewed the manuscript and carried out data analysis. All authors read and approved the final manuscript.

\section{Ethics}

The authors confirm that the ethical policies of the journal, as noted on the journal's author guidelines page, have been adhered to. All relevant guidelines for the use of animals in scientific studies were followed.

\section{Data availability statement}

Sequence data is available in NCBI GenBank database under the accession numbers: OM527159-OM527160. The data that support the findings of this study are available from the corresponding author upon reasonable request.

\section{ORCID}

GF: 0000-0001-5297-9036

ÉSZ: 0000-0002-2848-1577

GET: 0000-0002-7201-9646

ZSL: 0000-0003-3116-4633

BZ: 0000-0002-3503-8901

ZSV: 0000-0002-0432-3654

GK: 0000-0001-9775-3065

\section{References}


Akyildiz, G., Bente, D., Keles, A. G., Vatansever, Z., \& Kar, S. (2021). High prevalence and different genotypes of Crimean-Congo hemorrhagic fever virus genome in questing unfed adult Hyalomma marginatum in Thrace, Turkey. Ticks and Tick-Borne Diseases ,12 (2), 101622.

Arnaudov, A., Mikov, A., \& Georgiev, D. (2022). Infestation of the road-killed Eastern European hedgehogs (Erinaceus roumanicus) with Ixodidae ticks in some parts of Upper Thracian Plain (Bulgaria).ZooNotes , 192, $1-4$.

Arteaga, L. M., Bellido, J. L. M., Negredo, A. I., Criado, J. G., Lista, M. C. V., Serrano, J. Á. S., ... Belhassen-García, M. (2021). New circulation of genotype V of Crimean-Congo haemorrhagic fever virus in humans from Spain. PLOS Neglected Tropical Diseases ,15 (2), e0009197. https://doi.org/10.1371/journal.pntd.0009197

Bente, D. A., Forrester, N. L., Watts, D. M., McAuley, A. J., Whitehouse, C. A., \& Bray, M. (2013). Crimean-Congo hemorrhagic fever: History, epidemiology, pathogenesis, clinical syndrome and genetic diversity. Antiviral Research , 100 (1), 159-189. https://doi.org/10.1016/j.antiviral.2013.07.006

Brooks, D. R., Hoberg, E. P., \& Boeger, W. A. (2019). The Stockholm Paradigm: Climate Change and Emerging Disease . Chicago: University of Chicago Press.

Brooks, D. R., Hoberg, E. P., Boeger, W. A., Gardner, S. L., Galbreath, K. E., Herczeg, D., .. Dursahinhan, A. T. (2014). Finding Them Before They Find Us: Informatics, Parasites, and Environments in Accelerating Climate Change. Comparative Parasitology ,81 (2), 155-164. https://doi.org/10.1654/4724b.1

Capek, M., Literak, I., Kocianova, E., Sychra, O., Najer, T., Trnka, A., \& Kverek, P. (2014). Ticks of the Hyalomma marginatum complex transported by migratory birds into Central Europe. Ticks and Tick-Borne Diseases , 5 (5), 489-493. https://doi.org/10.1016/j.ttbdis.2014.03.002

Chitimia-Dobler, L., Schaper, S., Rieß, R., Bitterwolf, K., Frangoulidis, D., Bestehorn, M., ... Dobler, G. (2019). Imported Hyalomma ticks in Germany in 2018. Parasites \& Vectors ,12 (1), 134. https://doi.org/10.1186/s13071-019-3380-4

Ciloglu, A., Ibis, O., Yildirim, A., Aktas, M., Duzlu, O., Onder, Z., ... Inci, A. (2021). Complete mitochondrial genome characterization and phylogenetic analyses of the main vector of Crimean-Congo haemorrhagic fever virus: Hyalomma marginatum Koch, 1844. Ticks and Tick-Borne Diseases , 12 (5), 101736. https://doi.org/10.1016/j.ttbdis.2021.101736

Duscher, G. G., Hodžić, A., Hufnagl, P., Wille-Piazzai, W., Schötta, A.-M., Markowicz, M. A., ... Allerberger, F. (2018). Adult Hyalomma marginatum tick positive for Rickettsia aeschlimannii in Austria, October 2018. Eurosurveillance, 23 (48), 1800595. https://doi.org/10.2807/1560-7917.ES.2018.23.48.1800595

Eisen, L., \& Eisen, R. J. (n.d.). Benefits and Drawbacks of Citizen Science to Complement Traditional Data Gathering Approaches for Medically Important Hard Ticks (Acari: Ixodidae) in the United States.Journal of Medical Entomology . https://doi.org/10.1093/jme/tjaa165

Estrada-Peña, A., DAmico, G., \& Fernández-Ruiz, N. (2020). Modelling the potential spread of Hyalomma marginatum ticks in Europe by migratory birds. International Journal for Parasitology . https://doi.org/10.1016/j.ijpara.2020.08.004

Estrada-Peña, A., Mihalca, A. D., \& Petney, T. N. (2018). Ticks of Europe and North Africa: A Guide to Species Identification (1st ed. 2017 edition). New York, NY: Springer.

Földes, F., Madai, M., Németh, V., Zana, B., Papp, H., Kemenesi, G., .. Jakab, F. (2019). Serologic survey of the Crimean-Congo haemorrhagic fever virus infection among wild rodents in Hungary. Ticks and Tick-Borne Diseases , 10 (6), 101258. https://doi.org/10.1016/j.ttbdis.2019.07.002

Földvári, G., Márialigeti, M., Solymosi, N., Lukács, Z., Majoros, G., Kósa, J. P., \& Farkas, R. (2007). Hard Ticks Infesting Dogs in Hungary and their Infection with Babesia and Borrelia Species.Parasitology Research 
, 101 (S1), 25-34. https://doi.org/10.1007/s00436-007-0608-6

Földvári, G, Rigó, K., Jablonszky, M., Biró, N., Majoros, G., Molnár, V., \& Tóth, M. (2011). Ticks and the city: Ectoparasites of the Northern white-breasted hedgehog (Erinaceus roumanicus) in an urban park. Ticks and Tick-Borne Diseases , 2 (4), 231-234. https://doi.org/10.1016/j.ttbdis.2011.09.001

Gale, P., Stephenson, B., Brouwer, A., Martinez, M., de la Torre, A., Bosch, J., .. Muñoz, M. j. (2012). Impact of climate change on risk of incursion of Crimean-Congo haemorrhagic fever virus in livestock in Europe through migratory birds. Journal of Applied Microbiology, 112 (2), 246-257. https://doi.org/10.1111/j.13652672.2011.05203.x

Grandi, G., Chitimia-Dobler, L., Choklikitumnuey, P., Strube, C., Springer, A., Albihn, A., ... Omazic, A. (2020). First records of adult Hyalomma marginatum and H. rufipes ticks (Acari: Ixodidae) in Sweden. Ticks and Tick-Borne Diseases , 101403. https://doi.org/10.1016/j.ttbdis.2020.101403

Hansford, K. M., Carter, D., Gillingham, E. L., Hernandez-Triana, L. M., Chamberlain, J., Cull, B., ... Medlock, J. M. (2019). Hyalomma rufipes on an untraveled horse: Is this the first evidence of Hyalomma nymphs successfully moulting in the United Kingdom? Ticks and Tick-Borne Diseases , 10 (3), 704-708. https://doi.org/10.1016/j.ttbdis.2019.03.003

Hornok, S, Csörgő, T., de la Fuente, J., Gyuranecz, M., Privigyei, C., Meli, M. L., .. Hofmann-Lehmann, R. (2013). Synanthropic birds associated with high prevalence of tick-borne rickettsiae and with the first detection of Rickettsia aeschlimannii in Hungary. Vector Borne and Zoonotic Diseases (Larchmont, N.Y.), 13 (2), 77-83. https://doi.org/10.1089/vbz.2012.1032

Hornok, S., Flaisz, B., Takács, N., Kontschán, J., Csörgô, T., Csipak, Á., .. Kováts, D. (2016). Bird ticks in Hungary reflect western, southern, eastern flyway connections and two genetic lineages of Ixodes frontalis and Haemaphysalis concinna. Parasites $\&$ Vectors ,9 , 101. https://doi.org/10.1186/s13071-016-1365-0

Hornok, S, \& Horváth, G. (2012). First report of adult Hyalomma marginatum rufipes (vector of CrimeanCongo haemorrhagic fever virus) on cattle under a continental climate in Hungary. Parasites $\&$ Vectors , 5 , 170. https://doi.org/10.1186/1756-3305-5-170

Horváth, L. B. (1976). Precipitating antibodies to Crimean haemorrhagic fever virus in human sera collected in Hungary. Acta Microbiologica Academiae Scientiarum Hungaricae, 23 (4), 331-335.

Horváth, S. (1974). [Crimean hemorrhagic fever antibodies in Hungary]. Orvosi Hetilap , 115 (21), 1214.

Hubálek, Z., Sedláček, P., Estrada-Peña, A., Vojtíšek, J., \& Rudolf, I. (2020). First record of Hyalomma rufipes in the Czech Republic, with a review of relevant cases in other parts of Europe. Ticks and Tick-Borne Diseases , 11 (4), 101421. https://doi.org/10.1016/j.ttbdis.2020.101421

Jones, K. E., Patel, N. G., Levy, M. A., Storeygard, A., Balk, D., Gittleman, J. L., \& Daszak, P. (2008). Global trends in emerging infectious diseases. Nature , 451 (7181), 990-993. https://doi.org/10.1038/nature06536

Lang, J., Shan, Y., Zhang, M., Liu, J., \& Wang, F. (2022). The complete mitochondrial genome of Hyalomma rufipes (Acari: Ixodidae) from China and comparative analysis of mitogenomes in genus Hyalomma.International Journal of Acarology , 0 (0), 1-11. https://doi.org/10.1080/01647954.2022.2030794

Lesiczka, P. M., Daněk, O., Modrý, D., Hrazdilová, K., Votýpka, J., \& Zurek, L. (2022). A new report of adult Hyalomma marginatum and Hyalomma rufipes in the Czech Republic. Ticks and Tick-Borne Diseases ,13 (2), 101894. https://doi.org/10.1016/j.ttbdis.2021.101894

Lv, J., Wu, S., Zhang, Y., Chen, Y., Feng, C., Yuan, X., .. L Lin, X. (2014). Assessment of four DNA fragments (COI, 16S rDNA, ITS2, 12S rDNA) for species identification of the Ixodida (Acari: Ixodida).Parasites 8 Vectors , 7 (1), 93. https://doi.org/10.1186/1756-3305-7-93 
MacPhail, V. J., \& Colla, S. R. (2020). Power of the people: A review of citizen science programs for conservation. Biological Conservation, 249 , 108739. https://doi.org/10.1016/j.biocon.2020.108739

Magyar, N., Kis, Z., Barabás, É., Nagy, A., Henczkó, J., Damjanova, I., . . Pályi, B. (2021). New geographical area on the map of Crimean-Congo hemorrhagic fever virus: First serological evidence in the Hungarian population. Ticks and Tick-Borne Diseases ,12 (1), 101555. https://doi.org/10.1016/j.ttbdis.2020.101555

McGinley, L., Hansford, K. M., Cull, B., Gillingham, E. L., Carter, D. P., Chamberlain, J. F., ... Medlock, J. M. (2021). First report of human exposure to Hyalomma marginatum in England: Further evidence of a Hyalomma moulting event in north-western Europe? Ticks and Tick-Borne Diseases , 12 (1), 101541. https://doi.org/10.1016/j.ttbdis.2020.101541

Molnár, E. (1982). Occurrence of tick-borne encephalitis and other arboviruses in Hungary. Geographica Medicina , 12,78 .

Moraga-Fernández, A., Ruiz-Fons, F., Habela, M. A., Royo-Hernández, L., Calero-Bernal, R., Gortazar, C., ... Fernández de Mera, I. G. (2021). Detection of new Crimean-Congo haemorrhagic fever virus genotypes in ticks feeding on deer and wild boar, Spain.Transboundary and Emerging Diseases , 68 (3), 993-1000. https://doi.org/10.1111/tbed.13756

Németh, V., Oldal, M., Egyed, L., Gyuranecz, M., Erdélyi, K., Kvell, K., ... Jakab, F. (2013). Serologic evidence of Crimean-Congo hemorrhagic fever virus infection in Hungary. Vector Borne and Zoonotic Diseases (Larchmont, N.Y.) , 13 (4), 270-272. https://doi.org/10.1089/vbz.2012.1011

Nosek, J., Kožuch, O., \& Lysý, J. (1982). The finding of the female Hyalomma marginatum Koch, 1844 in southern Slovakia. Folia Parasitol. , 29 , 251. Scopus. Retrieved from Scopus.

Országos Epidemiológiai Központ. (2008). Krími-Kongó haemorrhagiás láz Görögországban. Epinfo , 15 (27), 313-315.

Rudolf, I., Kejíková, R., Vojtíšek, J., Mendel, J., Peňázziová, K., Hubálek, Z., ... Estrada-Peña, A. (2021). Probable overwintering of adult Hyalomma rufipes in Central Europe. Ticks and Tick-Borne Diseases , 12 (4), 101718. https://doi.org/10.1016/j.ttbdis.2021.101718

Sánchez-Seco, M. P., Sierra, M. J., Estrada-Peña, A., Valcárcel, F., Molina, R., Arellano, E. R. de, ... Negredo, A. (n.d.).Widespread Detection of Multiple Strains of Crimean-Congo Hemorrhagic Fever Virus in Ticks, Spain-Volume 28, Number 2-February 2022-Emerging Infectious Diseases journal-CDC . 28 (2), 394-402. https://doi.org/10.3201/eid2802.211308

Tamura, K., Stecher, G., \& Kumar, S. (2021). MEGA11: Molecular Evolutionary Genetics Analysis Version 11. Molecular Biology and Evolution , 38 (7), 3022-3027. https://doi.org/10.1093/molbev/msab120

Tran, T., Porter, W. T., Salkeld, D. J., Prusinski, M. A., Jensen, S. T., \& Brisson, D. (2021). Estimating disease vector population size from citizen science data. Journal of The Royal Society Interface, 18 (184), 20210610. https://doi.org/10.1098/rsif.2021.0610

Uiterwijk, M., Ibáñez-Justicia, A., van de Vossenberg, B., Jacobs, F., Overgaauw, P., Nijsse, R., ... Sprong, H. (2021). Imported Hyalomma ticks in the Netherlands 2018-2020. Parasites \&6 Vectors ,14 (1), 244. https://doi.org/10.1186/s13071-021-04738-x

Valcárcel, F., González, J., González, M. G., Sánchez, M., Tercero, J. M., Elhachimi, L., .. Olmeda, A. S. (2020). Comparative Ecology of Hyalomma lusitanicum and Hyalomma marginatum Koch, 1844 (Acarina: Ixodidae). Insects , 11 (5), 303. https://doi.org/10.3390/insects11050303

Vial, L., Stachurski, F., Leblond, A., Huber, K., Vourc'h, G., René-Martellet, M., ... Estrada-Peña, A. (2016). Strong evidence for the presence of the tick Hyalomma marginatum Koch, 1844 in southern continental France. Ticks and Tick-Borne Diseases , 7 (6), 1162-1167. https://doi.org/10.1016/j.ttbdis.2016.08.002 
Walker, A., Bouattour, A., Camicas, J. L., Estrada-Peña, A., Horak, I., Latif, A., .. Preston, P. M. (2003). Ticks of Domestic Animals in Africa: A guide to identification of species . Edinburgh: Bioscience Reports.

Wallménius, K., Barboutis, C., Fransson, T., Jaenson, T. G., Lindgren, P.-E., Nyström, F., ... Nilsson, K. (2014). Spotted fever Rickettsia species in Hyalomma and Ixodes ticks infesting migratory birds in the European Mediterranean area. Parasites \& Vectors ,7 (1), 318. https://doi.org/10.1186/1756-3305-7-318

\section{Tables}

Table 1. Species, stage, sex and host of tick specimens received within the tick monitoring citizen science project between 15 April and 7 December 2021

\section{Figure legends}

Figure 1. Phylogenetic tree of 22 partial gene sequences of the mitochondrial cytochrome oxidase c I gene of Hyalomma marginatum. The tree was constructed with the Neighbour-Joining method using the Tamura 3 -parameter method to compute evolutionary distances. We applied the bootstrap test with 1000 replicates. Novel sequence data is highlighted with bold letters and an arrow.

Figure 2. Phylogenetic tree of 24 partial gene sequences of the mitochondrial cytochrome oxidase c I gene of Hyalomma rufipes. The tree was constructed with the Neighbour-Joining method using the Tamura 3parameter method to compute evolutionary distances. We applied the bootstrap test with 1000 replicates. Novel sequence data is highlighted with bold letters and an arrow.

Figure 3. Geographical distribution of the ticks received by the tick monitoring citizen science projecthttps://www.kullancsfigyelo.hu. Each dot represents a tick sent in by the civil participants of the research. 


\title{
Dermacentor spp.
}

\author{
Ixodes spp.
}

\section{Haemaphysalis spp.}

\section{Hyalomma spp.}

Figure 4. Historical published data about the occurrence ofHyalomma ticks and Crimean-Congo Haemorrhagic Fever Virus (CCHFV) in Hungary in chronological order.

1 CCHFV isolation in 1972 from two I. ricinus ticks in Veszprém county (Molnár, 1982)

2 CCHFV seropositive cattle and sheep in 1973 in Hajdú-Bihar county ,

3 CCHFV antibody in 17 human sera. One from a slaughterhouse worker in Budapest, all others from Hajdú-Bihar county (L. B. Horváth, 1976).

4 Human CCHF infection from unknown source in Baranya county in 2004 (Országos Epidemiológiai Központ, 2008)

5 CCHFV seropositive brown hares collected between 2008 and 2009 from near Dévaványa village,

6 An engorged Hyalomma marginatum nymph found on a Northern white-breasted hedgehog in 2009 in an urban park of Budapest (G Földvári et al., 2011)

7 Two Hy. rufipes males found on two cows in September 2011 in south-western Hungary ,

8 CCHFV seropositive wild rodents collected between 2011 and 2013 in the Mecsek Mountain region , 
9 Three Hy. marginatum (two larvae and a nymph) collected from an European robin at the Ócsa Ringing Station in 2011 and three Hy. rufipes nymphs from a common whitethroat (Sylvia communis) in 2014 (S Hornok et al., 2013; S. Hornok et al., 2016)

Red crosses indicate origin of CCHFV seropositive blood donors collected between 2008-2017 (Magyar et al., 2021)

Red dots indicate the origin of the two Hyalomma specimens of the present study.
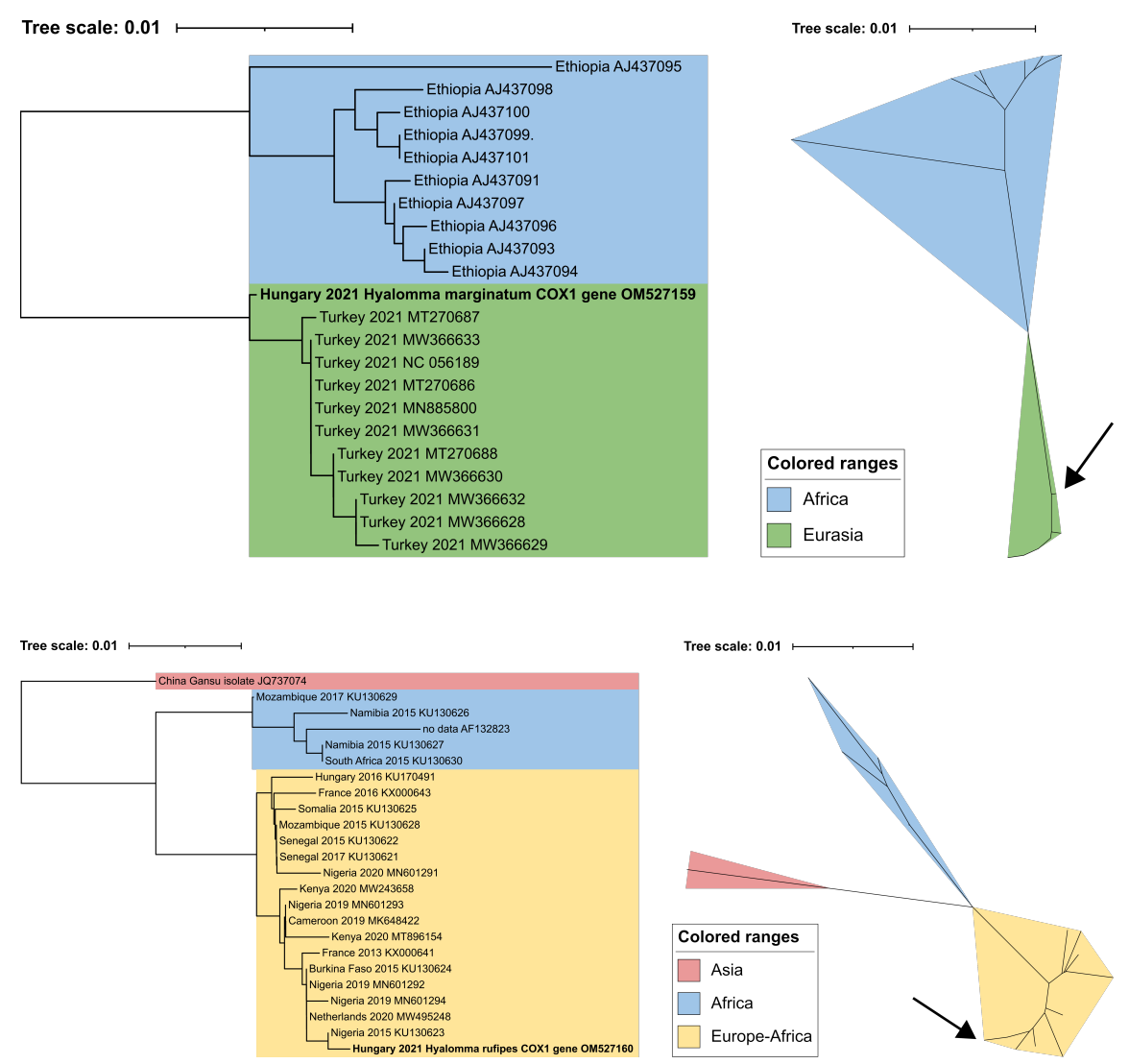

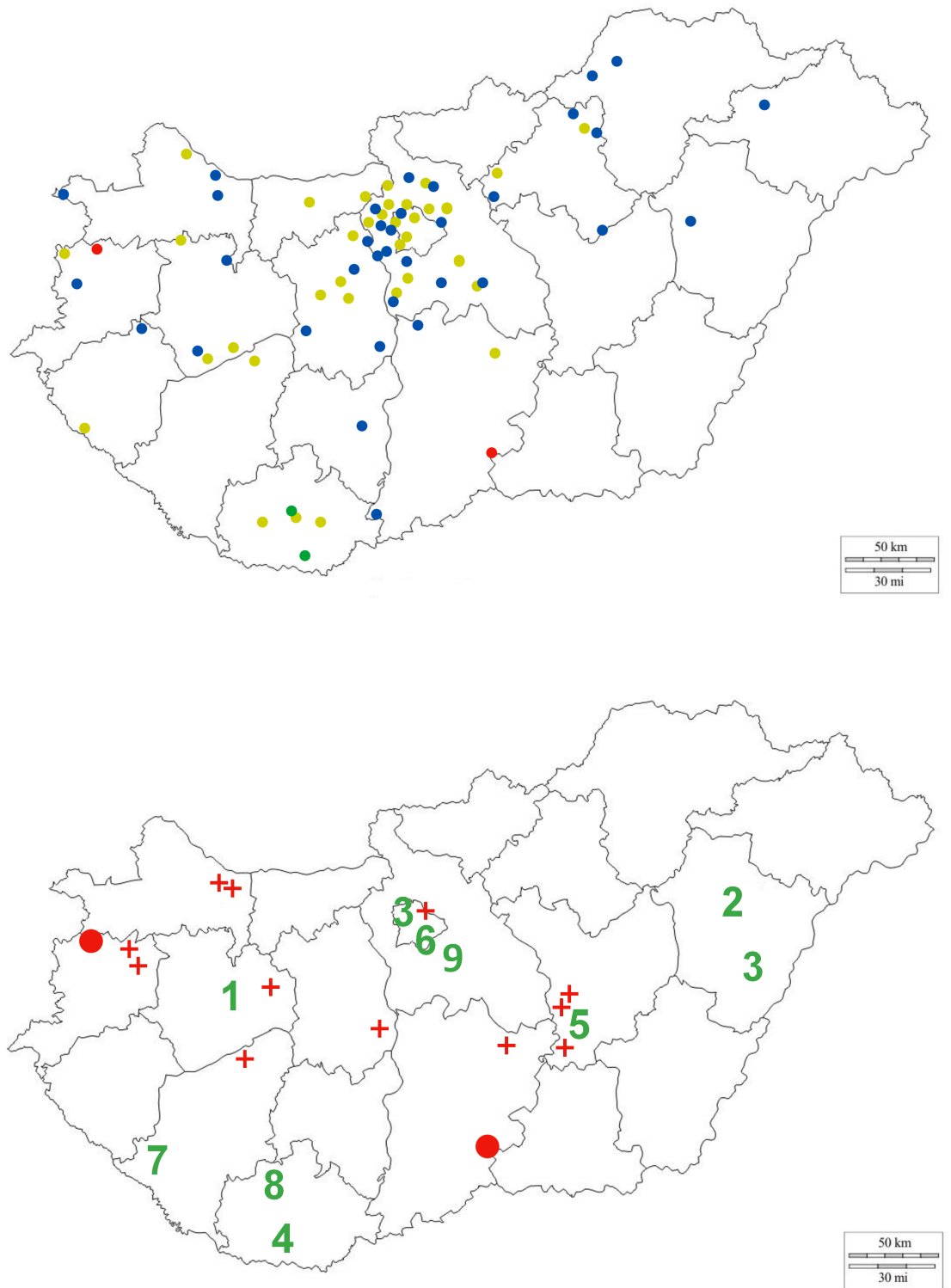

\section{Hosted file}

Table1.docx available at https://authorea.com/users/462203/articles/557693-emergenceof-hyalomma-marginatum-and-hyalomma-rufipes-adults-revealed-by-citizen-science-tickmonitoring-in-hungary 